\title{
Quantifying Treatment-Related Fluctuations in CIDP
}

Results of the GRIPPER Study

Jeffrey A. Allen, MD, Mamatha Pasnoor, MD, Mazen M. Dimachkie, MD, Senda Ajroud-Driss, MD, Thomas H. Brannagan, MD, Albert A. Cook, MD, Timothy Walton, MHS, CCRP, Mark B. Fiecas, PhD, John T. Kissel, MD, Ingemar Merkies, MD, Kenneth C. Gorson, MD, and Richard A. Lewis, MD

Correspondence Dr. Allen jaallen@umn.edu

Neurology ${ }^{\circledR}$ 2021;96:e1876-e1886. doi:10.1212/WNL.0000000000011703

\section{Abstract \\ Objective}

The objective of this study was to explore the extent of IV immunoglobulin (IVIG) treatmentrelated fluctuations (TRFs) by using home collection of daily grip strength in patients with chronic inflammatory demyelinating polyradiculoneuropathy (CIDP) and to use that information to develop evidence-based treatment optimization strategies.

\section{Methods}

This prospective observational study included 25 patients with well-defined CIDP. Participants recorded grip strength daily for 6 months. Disability and gait metrics were collected weekly. Serum immunoglobulin G levels were obtained at peak, trough, and midcycle IVIG intervals. Day-to-day grip strength changes $<10 \%$ were considered random. To identify patients with TRFs, 3-day averaged grip strength was calculated on each consecutive day after an IVIG infusion. TRFs were defined as $\geq 10 \%$ 3-day averaged grip strength difference compared to the pre-IVIG baseline.

\section{Results}

Participants successfully recorded grip strength on all but $9 \%$ of recordable days. Twelve patients (48\%) were classified as low/no fluctuaters and $13(52 \%)$ as frequent fluctuaters. In the frequent fluctuating group, grip strength improved over 1 week and thereafter was relatively stable until the third week after infusion. Grip strength was significantly correlated with measures of disability.

\section{Conclusions}

Grip strength collection by patients at home is reliable, valid, and feasible. A change in grip strength by $\geq 10 \%$ is a useful, practical, and evidence-based approach that may be used to identify clinically meaningful TRFs. From these data, we propose a treatment optimization strategy for patients with CIDP on chronic IVIG that may be applied to routine clinic care during both face-to-face and virtual video or telephone patient encounters.

\section{Trial Registration Information}

ClinicalTrials.gov Identifier: NCT02414490.

From the Department of Neurology (J.A.A.), and School of Public Heath (M.B.F.), Division of Biostatistics, University of Minnesota, Minneapolis; Department of Neurology (M.P., M.M.D.), University of Kansas Medical Center, Kansas City; Department of Neurology (S.A.-D.), Northwestern University, Chicago, IL; Department of Neurology (T.H.B.), Columbia University Medical Center, New York, NY; Neurology at Johns Creek (A.A.C.), LLC, Atlanta, GA; BriovaRx (T.W.), Lenexa, KS; Department of Neurology (J.T.K.), Ohio State University, Columbus; Department of Neurology (I.M.), Maastricht University Medical Centre+; Curaçao Medical Center (I.M.), Willemstad, the Netherlands; Department of Neurology (K.C.G.), St. Elizabeth's Medical Center, Tufts University School of Medicine, Boston, MA; and Department of Neurology (R.A.L.), Cedars-Sinai Medical Center, Los Angeles, CA.

Go to Neurology.org/N for full disclosures. Funding information and disclosures deemed relevant by the authors, if any, are provided at the end of the article. 


\section{Glossary}

CIDP = chronic inflammatory demyelinating polyradiculoneuropathy; COVID-19 = coronavirus disease 2019; GRIPPER = IVIg Treatment-Related Fluctuations in CIDP Patients Using Daily Grip Strength Measurements; I-RODS = Inflammatory Rasch-Built Overall Disability Scale; IgG = immunoglobulin G; IVIG = IV immunoglobulin; ONLS = Overall Neuropathy Limitations Scale; TRF = treatment-related fluctuation; TRF-d $=$ TRF deterioration; $\mathbf{T R F}-\mathbf{i}=$ TRF improvement; $\mathbf{T U G}=$ Timed Up and Go.

Chronic inflammatory demyelinating polyradiculoneuropathy (CIDP) is an acquired immune-mediated disorder of peripheral nerves with a heterogeneous clinical course. ${ }^{1}$ While all patients by definition must evolve over $\geq 8$ weeks, the course that follows can be monophasic, relapsing-remitting, or progressive. Fifteen percent of patients have a monophasic illness and are adequately treated with a single course of IV immunoglobulin (IVIG), ${ }^{2}$ and about one-third achieve a durable period of drug-free remission at some point during their illness. ${ }^{3}$ The remaining patients have persistent disease activity requiring long-term immunotherapy to prevent or minimize relapse and progression. The heterogeneity in the clinical course highlights the importance of periodic assessments of disease activity such that the treatment approach strikes a balance between minimizing immunotherapy exposure and maximizing treatment efficacy.

IVIG is one first-line CIDP treatment. While data from existing IVIG clinical trials are critically important to guide induction and early maintenance therapy, ${ }^{4}$ they are less informative when optimizing long-term treatment for individual patients. ${ }^{5}$ Current guidelines recommend using the lowest effective maintenance dose of IVIG, but the best strategy to achieve that end is unknown. ${ }^{1}$ In practice, IVIG infusion intervals as short as 7 to 14 days may be needed for optimal care of some patients, ${ }^{5}$ while in others, less frequent infusion intervals may achieve an equally satisfactory result.

Treatment-related fluctuations (TRFs) are the cyclic or periodic occurrence of clinical change at an interval after an IVIG infusion. TRFs may manifest as improvement after treatment (TRF-improvement [TRF-i]) or may be appreciated as deterioration at the end of an infusion cycle (TRF deterioration [TRF-d]). The objective of this study is to determine the extent of TRFs in CIDP patients treated with IVIG and to understand the feasibility of collecting these data in the home setting by patients and trained infusion nurses with the intent of using this information to improve CIDP treatment optimization strategies.

\section{Methods}

\section{Study Design}

IVIg Treatment-Related Fluctuations in CIDP Patients Using Daily Grip Strength Measurements (GRIPPER) is a prospective observational study conducted at 5 centers in the United States between June 2015 and December 2018. Each participant enrolled by a site investigator (J.A.A., M.P., M.M.D., S.A.-D., A.C., T.H.B.) required diagnostic confirmation by an independent panel of 3 CIDP experts (J.T.K., R.A.L., K.C.G.). Deidentified clinical notes, nerve conduction studies, and pertinent laboratory data were reviewed by the panel. Patients in whom a diagnosis of definite or probable $\mathrm{CIDP}^{1}$ was not confirmed by majority vote were withdrawn from further study participation. Diagnostically confirmed patients proceeded with study activities. Each patient participated in the study for 6 months.

\section{Standard Protocol Approvals, Registrations, and Patient Consents}

This study (NCT02414490) was approved by the institutional review boards of the participating centers. Written informed consent was obtained from each participant before any study-specific activities.

\section{Participants}

Patients 18 to 85 years with a diagnosis of definite or probable CIDP as defined by European Federation of Neurological Societies/Peripheral Nerve Society diagnostic criteria were eligible. ${ }^{1}$ The dose and frequency of IVIG administration were at the discretion of the treating physician, but for the patient to be study eligible, the IVIG interval was required to be between 21 and 42 days for at least 3 months. Patients receiving pulse corticosteroids, subcutaneous immunoglobulin, and plasma exchange were excluded so as not to obscure IVIG-related TRFs. Other concomitant immunomodulating medications were allowed provided that dosing had been stable for 3 months before enrollment and no dose changes were anticipated during the study period. Additional key inclusion criteria included a CIDP Disease Activity Scale ${ }^{3}$ score of 3 (stable active) or 4 (improvement) and an Overall Neuropathy Limitations Scale (ONLS) upper limb disability score of $\geq 2$ at some time during the disease.

\section{Study Outcomes Assessments}

After completion of an initial screening visit, no additional institutional study site visits were performed. All study data were collected at the participant's home by the participant or by trained home infusion nursing personnel. Training protocols developed by the principal investigator (J.A.A.) and study project manager (T.W.) using standard data collection methods and procedures were used to train nurses in a standardized manner across all study sites. Daily patientcollected grip strength was recorded in a standard paper diary supplied to each patient. Weekly nursing assessments were 
entered by the nurse directly into a Health Insurance Portability and Accountability Act-compliant database using an iPad. Visiting study nurses reviewed patient diaries for completion and accuracy and transposed grip strength measurements from the diary to the database using an iPad. Study investigators were able to access the database for content review and data analysis. Nurses required completion of competency assessments before collection of study data. Nurse evaluators were trained by the principal investigator or project manager in accordance with methodology described for each specific outcome ${ }^{6-8}$ and as outlined in the study training manual. Competency assessments were repeated yearly. Participants were trained in person by the nurse using verbal and written instructions. Participant competency was assessed by the nurse at each in-person study visit.

Grip strength was obtained daily from both hands for 6 months by each participant using a Jamar dynamometer (JLW Instruments, Chicago, IL). The daily grip strength value was determined by taking the average of 3 recordings. Inflammatory Rasch-Built Overall Disability Scale (I-RODS), ${ }^{6}$ ONLS, Timed Up and Go (TUG), ${ }^{7}$ and modified Fatigue Severity Scale ${ }^{8}$ scores were collected weekly at in-home study visits for 6 months by a study nurse. Blood draws were performed by the study nurse at 3 time points surrounding IVIG infusions. The trough serum IgG level was collected immediately before IVIG infusion; a peak serum immunoglobulin G (IgG) level was collected 5 minutes after IVIG infusion; and a midcycle level was drawn 2 weeks after IVIG infusions.

\section{Approach to Defining TRFs}

To differentiate random grip strength changes (hereafter referred to as noise) from TRFs, we first wanted to know the degree of day-to-day grip strength fluctuation due to chance alone. The mean daily change in grip strength was calculated across all participants. We considered day-to-day grip strength changes in kilograms of $<10 \%$ on any 2 consecutive days as noise to be not clinically meaningful. A value of $10 \%$ was selected because it approximated 2 SDs of the mean daily fluctuation in grip strength and is an easier metric to apply during routine clinical care. We recognized that although dayto-day fluctuations $\geq 10 \%$ were infrequent, noise of this magnitude may still occur simply by chance. To better filter out random noise that happened to be $\geq 10 \%$, we next calculated 3-day averaged grip strength values for each consecutive day (target day, +1 day, -1 day) beginning on the second day after any given IVIG infusion. Baseline averages were recalculated at each IVIG infusion so that there was a continuously updated average by which to make comparisons within each IVIG infusion cycle. A cycle with a TRF was defined as TRF-i or TRF-d if there was a $\geq 10 \%$ change from baseline on $\geq 2$ consecutive 3 -day averaged calculations. The dominant and nondominant hands were analyzed separately. From these findings, participants were classified as frequent fluctuaters if TRFs (TRF-d or TRF-i) occurred in at least $50 \%$ of the total number of cycles in at least 1 hand. Participants not meeting this definition were classified as low/no fluctuaters. In both groups, time to reach $\geq 10 \%$ improvement, days per cycle $\geq 10 \%$ change, and frequency of TRF-i cycles that returned to baseline by the end of the cycle were recorded. Demographic data, treatment history, and serum IgG characteristics were also compared between groups.

\section{Statistical Analysis}

Descriptive statistics, including counts and percentages, were used for nominal or dichotomous variables. Categorical variables were analyzed with the Pearson $\chi^{2}$ and Fisher exact test. Comparison of patient-collected and nurse-collected grip strength was performed by a random-effects time series regression analysis of averaged daily grip strength. The relationship between TRFs and other outcome measures was explored by using a linear mixed model to assess the association between each measure and grip strength for each hand. Fixed effects in the model were used to adjust for both the number of weeks since the last infusion and the fluctuation group (frequent fluctuaters and low/no fluctuaters), and a random intercept was used to account for the repeated measures within an individual. An interaction term between the fluctuation group and the outcome measure under review was considered, but the resulting model did not yield improved fit to the data. Missing data were addressed with Multivariate Imputation by Chained Equations (R Foundation for Statistical Computing, Vienna, Austria) to create $\mathrm{m}=$ 20 imputed data sets. ${ }^{9}$ Model parameters were pooled across the imputed data sets to obtain a single set of estimates and standard errors. ${ }^{10}$ Model fit to the data was quantified with $R^{2}$ values for mixed models that were extracted from and then averaged across the imputed data sets, where $R^{2}$ represents strength of association between the variables. ${ }^{11}$ Statistical analyses were compiled with Stata 13.0 (StataCorp, College Station, TX), Microsoft Excel (Microsoft Corp, Redmond, $\mathrm{WA}$ ), and $\mathrm{R}$ ( $\mathrm{R}$ Core Team, version 3.5.0).

\section{Data Availability}

The datasets analyzed in the current study are not publicly available, but anonymized study data can be made available to qualified investigators from the corresponding author on reasonable request.

\section{Results}

\section{Study Population and Participant Disposition}

Thirty patients were screened for eligibility. Three participants did not meet CIDP diagnostic criteria after review by the expert panel and were excluded. Two additional participants met all criteria but withdrew before the baseline assessment for personal reasons, leaving 25 participants for data collection. A complete 6-month dataset was collected in 23 participants. Data collection was truncated at 6 and 7 weeks in 2 participants due to unexpected pregnancy and participant accessibility. Baseline demographic and treatment data are shown in table 1 . All but 1 of the participants were righthanded. The mean duration of IVIG exposure before study 
Table 1 Baseline Demographic, Outcomes, and Treatment Characteristics

\begin{tabular}{|c|c|}
\hline Characteristics $(n=25)$ & \\
\hline Age (SD, range), y & $53.6(13.9,22-76)$ \\
\hline Female, $\mathbf{n}(\%)$ & $14(56)$ \\
\hline \multicolumn{2}{|l|}{ Ethnicity, n (\%) } \\
\hline Black & $3(12)$ \\
\hline Asian & $2(8)$ \\
\hline Hispanic & $1(4)$ \\
\hline Middle Eastern & $1(4)$ \\
\hline White & $18(72)$ \\
\hline Weight (SD, range), kg & $87.6(23.23,54-154)$ \\
\hline BMI (SD, range), kg/m² & $31.4(7.83,19.3-50.4)$ \\
\hline Symptom duration (SD, range), mo & $71.5(77.7,5-276)$ \\
\hline Time since diagnosis (SD, range), mo & $53.5(72.2,3-270)$ \\
\hline $\begin{array}{l}\text { EFNS/PNS definite (panel } \\
\text { determination), } \mathrm{n}(\%)\end{array}$ & $20(80 \%)$ \\
\hline \multicolumn{2}{|l|}{ CDAS score, $\mathrm{n}(\%)$} \\
\hline 3 (stable active) & $19(76)$ \\
\hline 4 (improving) & $6(24)$ \\
\hline \multicolumn{2}{|l|}{ ONLS score } \\
\hline $\begin{array}{l}\text { Upper limb at study entry, mean } \\
\text { (SD, range) }\end{array}$ & $1.4(0.7,0-3)$ \\
\hline $\begin{array}{l}\text { Upper limb disease maximum, } \\
\text { mean (SD, range) }\end{array}$ & $2.9(0.8,2-5)$ \\
\hline $\begin{array}{l}\text { Total at disease entry, } \\
\text { mean (SD, range) }\end{array}$ & $2.5(1.53,1-6)$ \\
\hline I-RODS score, mean (SD, range) & $64.1(17.1,31-93)$ \\
\hline $\begin{array}{l}\text { Grip strength dominant hand, } \\
\text { mean (SD, range), kg }\end{array}$ & $26.0(11.4,8.2-47.2)$ \\
\hline $\begin{array}{l}\text { Grip strength nondominant hand, } \\
\text { mean (SD, range), kg }\end{array}$ & $24.3(10.7,7.3-42.6)$ \\
\hline mFSS score, mean (SD, range) & $13.0(7.68,0-21)$ \\
\hline TUG score, mean (SD, range), $s$ & $10.6(5.5,6.5-34.1)$ \\
\hline \multicolumn{2}{|l|}{ IVIG treatment history } \\
\hline Duration, mo (SD, range) & $29.2(43.7,3-192)$ \\
\hline Dose (SD, range), $\mathrm{g}$ & $80.6(25.95,45-155)$ \\
\hline Dose (SD, range), g/kg & $0.93(0.18,0.46-1.27)$ \\
\hline Treatment interval (SD, range), wk & $3.9(1.1,3-6)$ \\
\hline Dose per 3 wk (SD, range), g/kg & $0.75(0.25,0.28-1.27)$ \\
\hline \multicolumn{2}{|l|}{ Concomitant immunotherapy $(n=3)$} \\
\hline Prednisone: dose, duration & $20 \mathrm{mg}, 3 \mathrm{mo}$ \\
\hline Prednisone: dose, duration & $25 \mathrm{mg}, 8 \mathrm{mo}$ \\
\hline Mycophenolate: dose, duration & $1,000 \mathrm{mg}$ twice daily, $19 \mathrm{mo}$ \\
\hline
\end{tabular}

Abbreviations: $\mathrm{BMI}=$ body mass index; CDAS = CIDP Disease Activity Scale; EFNS/PNS = European Federation of Neurological Societies/Peripheral Nerve Society; I-RODS = Inflammatory Rasch-Built Overall Disability Scale; IVIG = IV immunoglobulin; mFSS = Modified Fatigue Severity Scale; ONLS = Overall Neuropathy Limitations Scale; TUG = Timed Up and Go. enrollment was 29.2 months, ranging from 3 to 192 months. The average IVIG dose per infusion was $0.93 \mathrm{~g} / \mathrm{kg}$. Per study inclusion criteria, treatment intervals ranged from 3 to 6 weeks (mean 3.9 weeks). When the dose of IVIG was adjusted to the amount received every 3 weeks, the calculated dose was only $0.75 \mathrm{~g} / \mathrm{kg}$. Only 3 patients were receiving an immunotherapy agent in addition to IVIG at the time of enrollment (table 1).

Across all participants, grip strength was recorded on 3,584 of a possible 3,938 days. Only 9\% of daily grip strength collection was missed during the study. Random-effects regression analysis revealed a very high correlation between nursecollected grip strength and patient-collected grip strength $\left(R^{2}\right.$ $=0.95$ ), indicating good external validity (figure 1).

\section{Differentiating Random Grip Strength Noise From TRFs}

The mean daily change in grip strength was 3.1\% (SD 3.5\%) in the dominant hand and 3.0\% (SD 3.3\%) in the nondominant hand. Of the 3,538 days available for analysis, $16.4 \%$ of dominant and $17.0 \%$ of nondominant consecutive days exceeded a $10 \%$ change. When 3 -day averaged grip strength was calculated, consecutive averaged days with a $>10 \%$ change was only $1.6 \%$ on the dominant hand and $1.5 \%$ on the nondominant hand.

When our definitions of fluctuation classification were used, 12 patients (48\%) were classified as low/no fluctuaters and 13 $(52 \%)$ as frequent fluctuaters (figure 2). TRFs were observed in $61.7 \%$ (dominant) and $69.1 \%$ (nondominant) of all IVIG cycles in the frequent group and only $\approx 20 \%$ of cycles in the low/no group (table 2). In the frequent fluctuating group, TRF-i (dominant 41.9\%, nondominant 46.4\%) occurred about twice as often as TRF-d (dominant 19.7\%, nondominant 19.7\%), whereas in the low/no fluctuating group, the frequencies of TRF-i and TRF-d were similar (all $\approx 10 \%)$. TRFs of the greatest physiologic interest were TRF-i that fell back to baseline by the end of the cycle (i.e., intracycle improvement and then decline temporally associated with IVIG infusions, sometimes referred to as wear-off) and TRF-d that continued to be $\geq 10 \%$ at the end of the cycle (i.e., intracycle decline that did not spontaneously recover before the subsequent infusion). In the frequent fluctuation group, this pattern was observed in almost $60 \%$ of TRF-i cycles and almost $70 \%$ of TRF-d cycles. Of the 3 patients who were receiving other immunotherapy in addition to IVIG, all 3 were stratified into the frequent TRF group.

\section{Temporal Association Between IVIG and TRFs}

To understand when TRFs occurred relative to any given IVIG infusion, 3-day averaged grip strength was calculated for each consecutive day after each IVIG infusion. Figure 3 illustrates the consecutive daily percent change in grip strength from the combined dominant and nondominant hands averaged across all patients and all cycles. The frequent fluctuating group showed a clear rise in 3-day averaged grip strength 

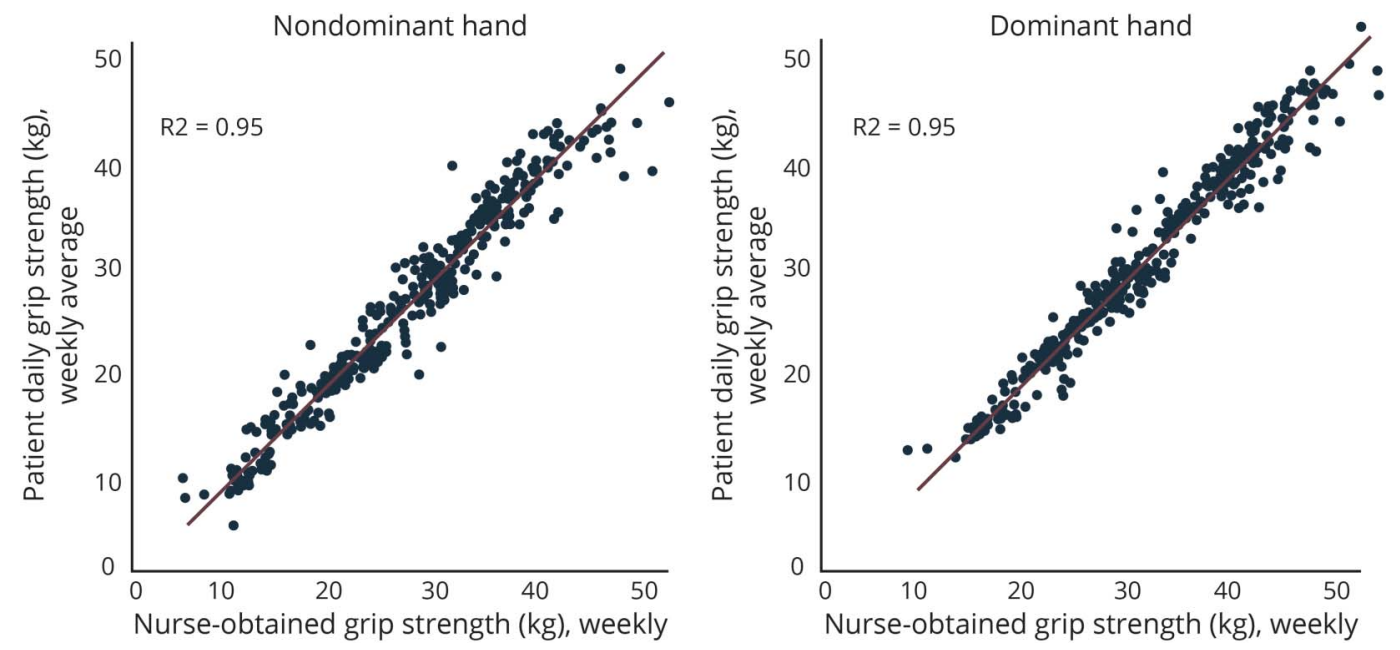

during the first week, followed by a period of relative stability over the second and third weeks, and then rapid return to baseline between the third and fourth weeks (figure 3A). No such pattern was appreciated in the low/no fluctuating group (figure 3B). Peak mean percent change in grip strength relative to the baseline infusion day across all cycles was $8.2 \%$ (dominant) and $14.4 \%$ (nondominant) in the frequent fluctuating group compared to $2.0 \%$ (dominant) and $3.1 \%$ (nondominant) in the group without frequent fluctuations ( $p$ $<0.01$ ). When 3-day averaged calculations were used, patients in the frequent fluctuating group spent an average of $26.9 \%$ (dominant) and $33.6 \%$ (nondominant) days during each

Figure 2 Dominant and Nondominant Hand TRFs in 25 Patients

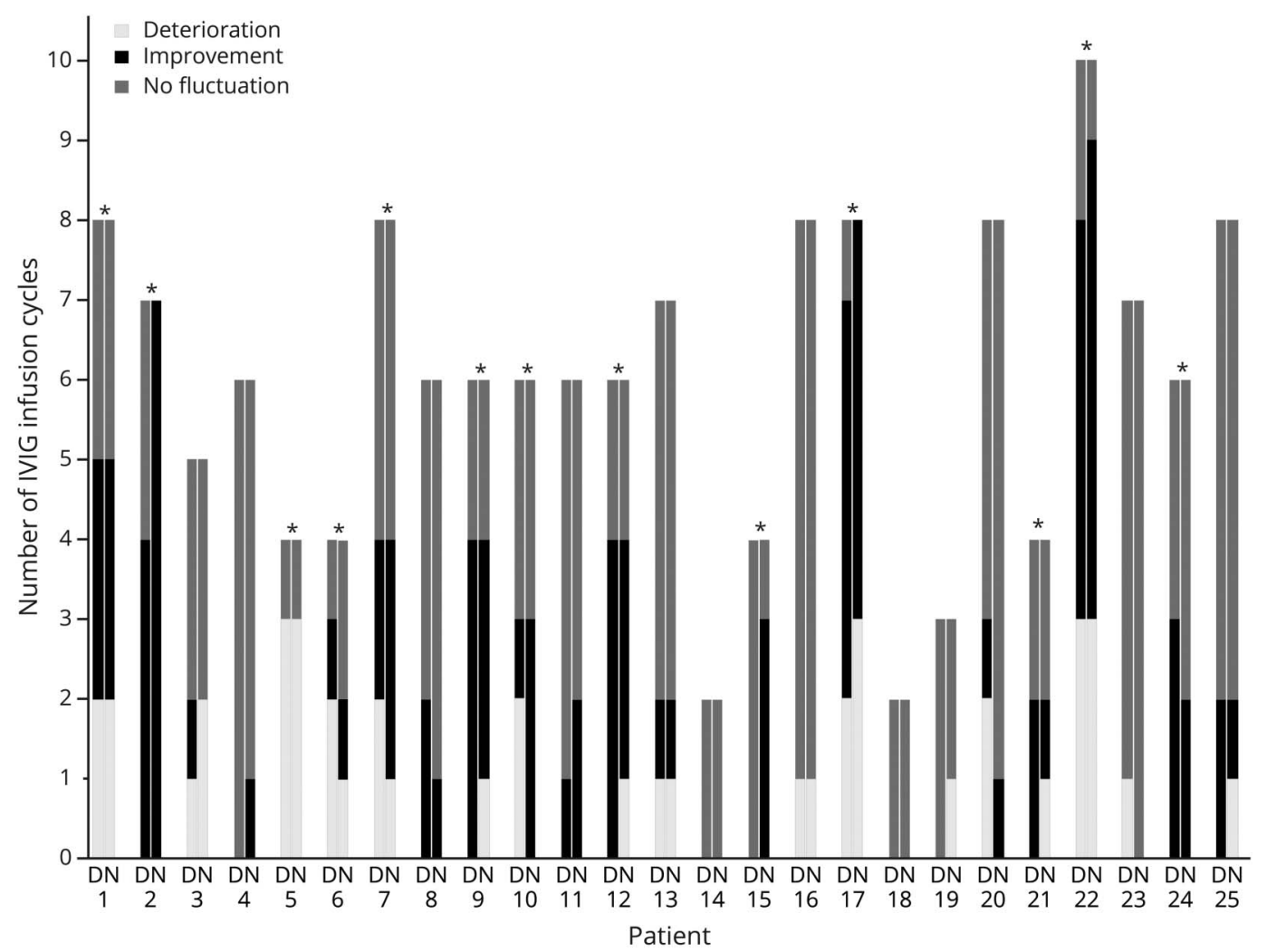

Treatment-related fluctuation (TRF) deterioration (white), TRF improvement (black), or no TRFs (gray) in patients receiving IV immunoglobulin (IVIG). D = dominant hand; $\mathrm{N}=$ nondominant hand. *Patients classified as frequent fluctuaters. 
Table 2 Occurrence of Dominant and Nondominant Hand TRFs in Patients Receiving IVIG

\begin{tabular}{|c|c|c|c|c|}
\hline & \multicolumn{2}{|c|}{ Frequent Fluctuaters $(n=13)$} & \multicolumn{2}{|c|}{ Low/No Fluctuaters $(n=12)$} \\
\hline & Dominant & Nondominant & Dominant & Nondominant \\
\hline Total number of IVIG cycles, $n$ & 81 & 81 & 68 & 68 \\
\hline IVIG cycles with $\geq 10 \%$ change, $n(\%)$ & $50(61.7)$ & $56(69.1)$ & $14(20.5)$ & $13(19.1)$ \\
\hline IVIG cycles with TRF-i $\geq 10 \%$ change, $n$ (\%) & $34(41.9)$ & $40(46.4)$ & $8(11.8)$ & $7(10.3)$ \\
\hline IVIG cycles with TRF-d $\geq 10 \%$ change, $n$ (\%) & $16(19.7)$ & $16(19.7)$ & $6(8.8)$ & $6(8.8)$ \\
\hline IVIG cycles with TRF-i that returned to baseline at end of cycle, $n$ (\%) & $20(58.8)$ & $22(55.0)$ & $4(50.0)$ & $4(57.1)$ \\
\hline IVIG cycles with TRF-d that remained $\geq 10 \%$ change at end of cycle, $n(\%)$ & $11(68.8)$ & $10(62.5)$ & $2(33.3)$ & $1(16.7)$ \\
\hline
\end{tabular}

Abbreviations: IVIG = IV immunoglobulin; TRF = treatment-related fluctuation; TRF-d = TRF deterioration; TRF-i = TRF improvement.

cycle of $\geq 10 \%$ baseline grip strength compared to $8.6 \%$ (dominant) and $5.6 \%$ (nondominant) of days in the low/no fluctuating group $(p<0.01)$.

\section{Grip Strength Variation by IVIG Dose, Interval, and IgG Level}

IVIG treatment intervals and dosing were similar in both groups (table 3). Patients with frequent TRFs carried a diagnosis of CIDP for a shorter duration than those without TRFs $(p=0.01)$. While patients with TRFs also tended to have a less extensive history of IVIG exposure, the difference was not statically significant. There were no differences in peak, trough, or midcycle IgG levels in the frequent vs low/no groups, nor were there differences in $\Delta \mathrm{IgG}$ level between trough and peak or trough and midcycle (table 3 ).

\section{Correlation of Grip Strength With Other Outcomes}

After adjustment for the number of weeks since last infusion and degree of fluctuation, grip strength was significantly correlated $(p<0.05)$ with I-RODS (positive correlation), ONLS (negative correlation), and TUG (negative correlation) scores but not modified Fatigue Severity Scale score ( $p=$ $0.28)$. Strength of correlations with I-RODS (nondominant

Figure 3 Percent Change in 3-Day Averaged Dominant and Nondominant (Combined) Hand Grip Strength

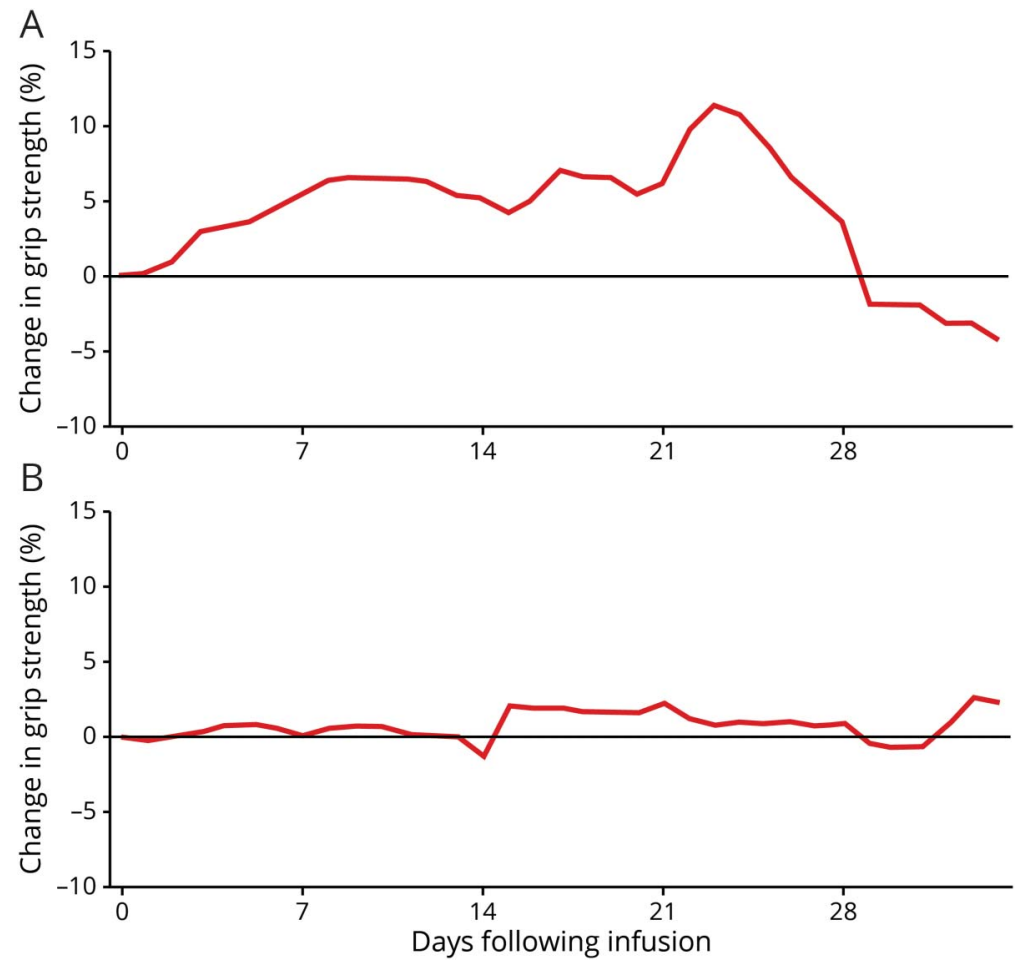

Grip strength across all patients and all cycles in participants with (A) frequent treatment-related fluctuations (TRFs) and (B) low or no TRFs. 
Table 3 IVIG Administration Characteristics and IgG Levels in Patients With and Without TRFs

\begin{tabular}{|c|c|c|c|}
\hline & Frequent Fluctuater $(n=13)$ & Low/No Fluctuater $(n=12)$ & $p$ Value \\
\hline IVIG dose, mean (SD, range), g/kg & $0.91(0.21,0.5-1.3)$ & $0.95(0.15,0.7-1.1)$ & 0.55 \\
\hline IVIG dose, mean (SD, range), g/kg per $21 \mathrm{~d}$ & $0.71(0.28,0.3-1.3)$ & $0.80(0.22,0.4-1.1)$ & 0.38 \\
\hline IVIG interval, mean (SD, range), wk & $4.2(1.2,3-6)$ & $3.8(0.9,3-6)$ & 0.35 \\
\hline Duration IVIG, mean (SD, range), mo & $18.5(18.1,3-55)$ & $40.8(59.4,3-192)$ & 0.21 \\
\hline Duration CIDP diagnosis, mean (SD, range), mo & $20.6(16.1,3-55)$ & $92.3(92.8,3-270)$ & 0.01 \\
\hline \multicolumn{4}{|l|}{ IgG level, mean (SD, range), mg/DL } \\
\hline Trough & $1,483(229,1,193-1896)$ & $1746(653,1,306-3,740)$ & 0.18 \\
\hline Midcycle & $1911(259,1,480-2,421)$ & $2,169(591,1,667-3,869)$ & 0.16 \\
\hline Peak & $3,706(867,2,344-5,133)$ & $4,001(680,2,939-5,242)$ & 0.36 \\
\hline$\Delta$ IgG trough to peak (SD, range), $\mathrm{mg} / \mathrm{DL}$ & $2,222(698,1,140-3,603)$ & $2,254(496,1,372-2,920)$ & 0.89 \\
\hline$\Delta \mathrm{IgG}$ trough to midcycle (SD, range), $\mathrm{mg} / \mathrm{DL}$ & $427(141,276-762)$ & $423(170,129-727)$ & 0.95 \\
\hline
\end{tabular}

Abbreviations: CIDP = chronic inflammatory demyelinating polyradiculoneuropathy; IgG = immunoglobulin G; IVIG = IV immunoglobulin; TRF = treatmentrelated fluctuation;

$R^{2}=0.231$, dominant $\left.R^{2}=0.199\right)$, ONLS $\left(R^{2}=0.164\right.$ both sides), and TUG (dominant $R^{2}=0.165$, nondominant $R^{2}=$ $0.162)$ scores showed no clear hand preference.

\section{Discussion}

This prospective observational study of patients with welldefined CIDP shows that daily grip strength collection by patients at home is reliable, valid, and feasible. We show that a $10 \%$ change in grip strength can be used to separate random noise from true TRFs, especially when 3-day averaged calculations are recorded. The observation that changes in grip strength correlate with measures of disability supports the notion that a $10 \%$ change in grip strength is clinically meaningful. In comparison, a fixed 8-kPa grip strength change on the Martin Vigorimeter device has been shown to discriminate between patients receiving treatment and those receiving placebo in CIDP clinical trials. ${ }^{12}$ Especially when rolling 5-day mean grip strength values are calculated, changes of $\geq 8 \mathrm{kPa}$ may be clinically meaningful and capable of distinguishing between treatment response and random day-to-day grip strength fluctuations. ${ }^{13}$ While both absolute and relative cutoffs may have a role in detecting a clinically meaningful change, we elected a percentage approach because it contextualizes the degree of change proportional to the patient's baseline strength. By using these parameters, we were able to identify a population of patients with frequent IVIGassociated TRFs and another population who were stable over multiple treatment cycles.

Our findings provide a template for the development of evidence-based treatment optimization strategies for patients with CIDP on long-term IVIG (figure 4). The process may begin by collecting and averaging 3 consecutive days of grip strength immediately before IVIG. This value would serve as the patient's baseline or trough grip strength. Rather than daily collection of grip strength as was performed in this study, a more feasible approach may be to collect grip strength a minimum of 3 consecutive $d$ / wk and use those values to obtain an averaged calculation. The frequency of grip strength collection may be increased at the clinician's discretion if closer surveillance is needed. Although collection of bilateral hand grip strength is minimally burdensome, considering that dominant and nondominant hands yielded similar results, data from just 1 hand may suffice. If only 1 hand is measured, care needs to be taken that the same hand is always recorded when assessing for change. Patients with 3-day averaged grip strength changes $<10 \%$ of baseline may be classified as stable, provided that there are also no changes in disability metrics, and hence may be candidates for IVIG reduction. Although disability assessments provide important information under any circumstance, they are particularly valuable within the context of well-documented grip strength TRFs. We propose that if 3-day averaged grip strength changes by $\geq 10 \%$ but there is no impact on disability, then no treatment modification may be needed because a minor degree of objective deterioration may provide assurance that treatment is both needed and not overused. This effectively would be considered optimized IVIG. Conversely, patients with grip strength TRFs that are accompanied by fluctuating or declining disability may be candidates for IVIG escalation or other immunotherapies in lieu of or in addition to IVIG. The duration of monitoring before a TRF determination can be made is at the discretion of the clinician, taking into account the unique circumstances of individual patients. However, considering that we observed TRFs in $60 \%$ to $70 \%$ of IVIG cycles in the 


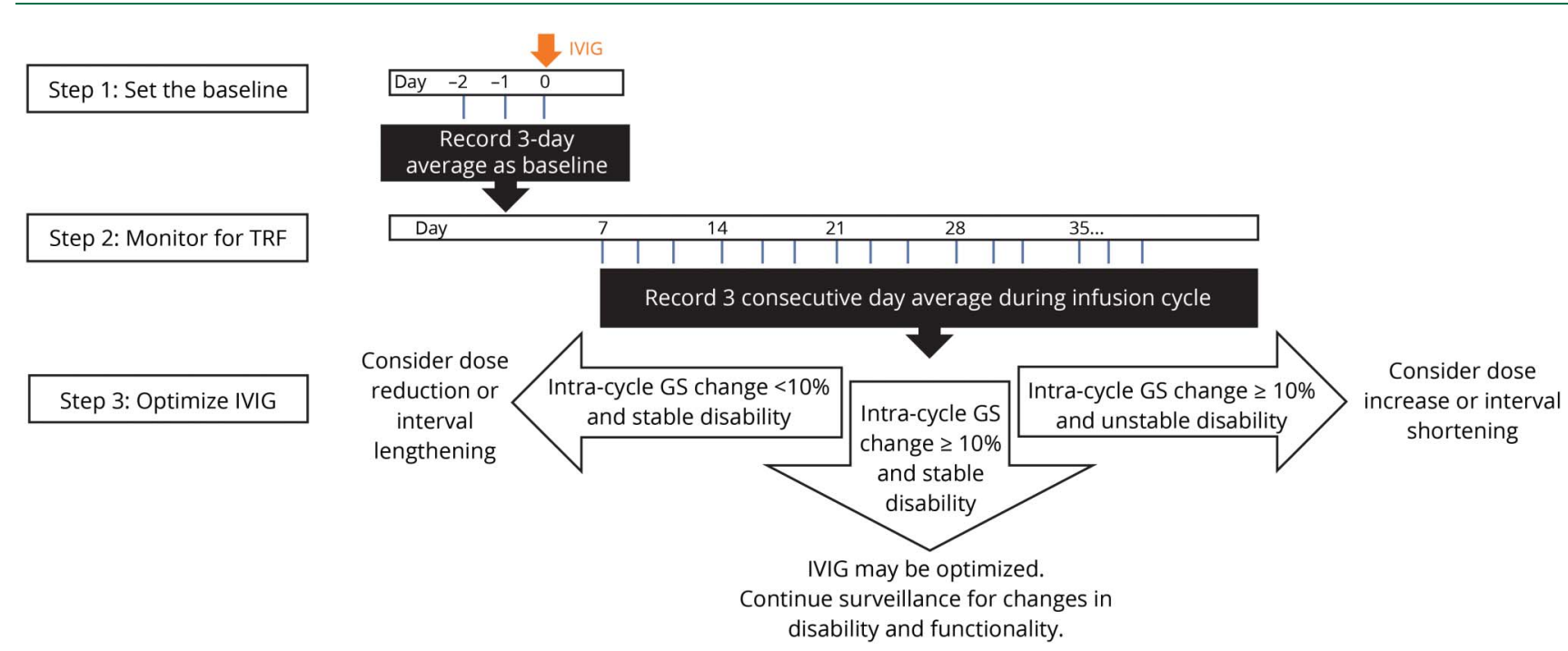

CIDP = chronic inflammatory demyelinating polyradiculoneuropathy; GS = grip strength; IVIG = IV immunoglobulin; TRF = treatment-related fluctuation.

frequent fluctuating group, in many cases, TRFs will be captured within the first monitored cycle.

IVIG optimization can be achieved by dose reduction or interval lengthening in stable patients or by the opposite approach in unstable patients. In clinical practice, immunoglobulin optimization includes consideration of health care access and patient convenience (which may favor less frequent but higher-dose administration) and IVIG tolerability (which may favor more frequent but lower-dose administration). Biological determinants also are important considerations. It is unknown whether optimal therapeutic efficacy is more strongly influenced by the use of high-dose IVIG to achieve a high peak serum $\operatorname{IgG}$ level or if more frequently administered IVIG at lower doses that finds a steady-state level with lower peaks and higher troughs is more desirable. ${ }^{5,14}$ In Guillain-Barré syndrome, patients who had a greater increase in the serum IgG level after a standard dose of IVIG had superior clinical outcomes compared to those with smaller changes in IgG level. ${ }^{15}$ Similar observations correlating grip strength to serum IgG concentration 1 week after infusion have been demonstrated in CIDP. ${ }^{16}$ We found no differences in the peak, trough, or midcycle IgG levels or in IgG level changes among patients with frequent TRFs and those without TRFs. From a practical perspective, these observations suggest that while a steady-state or peak IgG level may be needed to achieve a desirable effect, the required level is variable from patient to patient (or perhaps even variable in a single patient at different stages of disease), and this variability limits the usefulness of serum IgG level monitoring for making clinical decisions.

Our study has several important limitations. No outcome measure perfectly assesses every patient with CIDP. We cannot exclude the possibility that grip strength failed to capture TRFs in some patients. Patients with atypical CIDP variants ${ }^{1}$ who predominantly harbor lower limb or multifocal manifestations may be most at risk for errors of exclusion. Our requirement that all study participants have upper limb involvement (as assessed by upper limb ONLS score $\geq 2$ ) minimizes the likelihood that we failed to capture TRFs because of regional (i.e., nonhand) manifestations but also limits the degree to which our results may be generalized to some atypical variants. We also acknowledge that isolated grip strength changes may not reflect overall functionality in all patients. Grip strength has been shown to be a reliable measure of global neurologic status in patients with CIDP not just limited to upper limb or exclusively motor function. ${ }^{17,18}$ Consistent with those observations, we found that changes in grip strength correlated with measures of disability (I-RODS score) ${ }^{6}$ and gait impairment (TUG score). ${ }^{7}$ These findings support the premise that changes in grip strength likely reflect clinically relevant information not limited to hand function. Our novel methodology by which we differentiated frequent from low/no fluctuaters and by which we differentiated random noise from TRFs may also be questioned. A threshold of $50 \%$, although arbitrary, was chosen because it indicated fluctuations in more cycles than not and thus was a good way to separate frequent fluctuaters from low/no fluctuaters. Regarding random noise differentiation, while less precise than a more standard benchmark of 2 SD below the mean, ${ }^{19}$ a value of $10 \%$ was used because it closely approximated 2 SD of the mean daily fluctuation in grip strength and is an easier metric to apply during routine clinical care. With this approach, only $16 \%$ to $17 \%$ of consecutive days had changes $\geq 10 \%$, and when 3 -day averaged calculations were performed, the occurrence of consecutive day changes by $>10 \%$ dropped to $\approx 1.5 \%$. We feel that a $10 \%$ threshold is therefore a useful and practical 
approach to define TRFs. Unlike an absolute 8-kPa cutoff, which impractically uses the same benchmark for patients regardless of their baseline strength, a percentile threshold is more adaptable to different patients with variable degrees of strength impairment and to the same patient at different stages or severities of disease. An important caveat with both the percentile and absolute criterion is the difficulty of interpreting grip strength changes in patients with very weak grip, in which context neither a $10 \%$ nor an 8 -kPa change in grip strength may be meaningful. In our study, 2 patients in the frequent fluctuating cohort and 1 patient in the low/no fluctuating group had baseline grip strength values of $\approx 10 \mathrm{~kg}$. Although we did not exclude patients with this magnitude of grip strength impairment from our study, we advise caution during routine clinical care when interpreting grip strength changes when baseline strength is $<10 \mathrm{~kg}$.

The coronavirus disease 2019 (COVID-19) pandemic has immediately affected the way that health care providers interact with patients. While some of the adaptions may be relaxed as testing, treatment, and vaccination for COVID19 become available, the need and desire to evaluate and monitor disease states remotely may outlast the pandemic. Some modalities are easier to capture remotely than others. Strength impairment assessments typically rely on person-to-person contact for Medical Research Council scoring and hence are challenging to quantify through a telephone or video encounter. Grip strength monitoring by patients at home may fill the gap between what can be done face to face in clinic and what is often recorded during distanced evaluations. Recognizing that CIDP disease monitoring is best done from a multimodality approach, we also encourage collection of disability outcomes concomitantly with grip strength. ${ }^{20}$ Although some training is required, the I-RODS and ONLS (as well as the closely related Inflammatory Neuropathy Cause and Treatment scale ${ }^{21}$ ) are easy to learn and equally as feasible for patients to complete at home on their own. Strength assessment with handheld dynamometer and disability assessment with one of the validated patient-reported disability scales are well poised to objectify disease progression and treatment response in patients with CIDP even without an inperson encounter. Logistically, there are challenges. There is a need to improve accessibility to grip strength recording devices before frequent home collection can be widely adopted to routine clinical care. Although the purchase price of handheld dynamometer (generally between US $\$ 150$ and $\$ 400$ ) is on par with other devices routinely used in the neurologic clinic, when considering home use for individual patients, the cost is not trivial. Documentation of data collected by patients at home also requires a process by which the findings can be seamlessly and rapidly communicated to the physician. Mobile applications that feed outcomes data directly into the patients' medical record may provide the optimal solution. We encourage partnerships with hospital administration, nonprofit health care collaborators, insurers, specialty pharmacies, and industry such that practical technological and fiscal solutions to these barriers can be overcome.

In the absence of a biomarker that accurately reflects CIDP disease activity, treatment optimization will foreseeably remain a process driven by clinical metrics. Failing to objectify treatment response (or absence of response) is one factor that contributes to both the overtreatment and overdiagnosis of CIDP, as well as to delayed treatment escalation for patients without benefit to first-line therapies. ${ }^{22}$ We strongly encourage the use of grip strength or other objective clinical outcome measures to justify escalation or de-escalation of immunotherapy because changes in these outcomes presumptively reflect an underlying biological process that cannot be directly measured. IVIG doses and treatment intervals required to achieve and maintain maximum clinical benefit may vary greatly between individuals and probably within any one individual at different stages of the disease. ${ }^{23}$ While such heterogeneity obfuscates evidence-driven treatment protocols, it should not obviate the need to use them to make informed treatment decisions. Herein, an approach is illustrated that has been derived from prospectively obtained data that can be used during routine clinical care and does not require inperson, physician-patient encounters. Future study is needed to understand whether this proposed approach leads to improved patient outcomes and more efficient IVIG use.

\section{Acknowledgment}

The authors would like to thank David Schaefer and Briova Rx infusion services (formerly AxelaCare Health Solutions, LLC) nursing staff for their participation in the project. We also thank John Ney (Department of Neurology, Boston University, Boston, MA) who assisted with a portion of the statistical analysis. The authors would like to thank CSL Behring, and in partiuclar Melvin Berger, Patty Riley, and Ann Leon for their support, without which this study could not have been successful.

\section{Study Funding}

This study was funded by an investigator-initiated grant provided by CSL Behring.

\section{Disclosure}

J.A. Allen has provided consulting services to Akcea, Alexion, Argenx, Momenta, CSL Behring, and Biotest. M. Pasnoor has provided consulting services to TerumboCT, Alexion, CSL Behring, Argenx, Momenta, and Catalalyst. M.M. Dimachkie has provided consulting services to ArgenX, Catalyst, CSLBehring, Kezar, Momenta, NuFactor, Octapharma, RMS Medical, Sanofi Genzyme, Shire Takeda, and Spark Therapeutics. M.M. Dimachkie received grants from Alexion, Alnylam Pharmaceuticals, Amicus, Biomarin, Bristol-Myers Squibb, Catalyst, CSL-Behring, Food and Drug Administration/Officeof Orphan Products Development, GlaxoSmithKline, Genentech, Grifols, Mitsubishi Tanabe Pharma, Muscular Dystrophy Association, NIH, Novartis, Sanofi Genzyme, Octapharma, Orphazyme, Sarepta Therapeutics, 
Shire Takeda, Spark, UCB Biopharma, Viromed/Healixmith, and TMA. S. Ajroud-Driss has provided consulting services to Alnylam Pharmaceutical, Akcea Therapeutics, MT Pharma, Biohaven, and Biogen. T. Brannagan has provided consulting services to Akcea, Alnylam, CSL-Behring, Grifols, Ionis, and Pfizer and has research support from Acceleron, Alnylam, Grifols, Pharnext, Ionis, Takeda, UCB Biopharma, and Viromed/Helixmith. A.A. Cook has provided consulting services to CSL Behring. M.B. Fiecas, T. Walton, and J.T. Kissel report no disclosures. I. Merkies has provided consulting services to CSL Behring, Grifols, UCB Pharma, Octapharma, LFB, and Argenx, all outside the scope of the current study. K.C Gorson has provided consulting services to CSL Behring, Shire, Grifols, UCB Pharma, Annexon, Argenx, Bioverativ, Pfizer, and Immunovant. R.A. Lewis has provided consulting services to CSL Behring, Shire, Annexon, Biotest, Pharnext, Alnylam, Akcea, Argenx, Momenta, Sanofi, and Pfizer. Go to Neurology.org/N for full disclosures.

\section{Publication History}

Received by Neurology September 11, 2020. Accepted in final form January 4, 2021.

Appendix Authors

\begin{tabular}{lll}
\hline Name & Location & Contribution \\
\hline Jeffrey A. & Department of Neurology, & Protocol design, site \\
Allen, MD & University of Minnesota, & $\begin{array}{l}\text { investigator, data } \\
\text { Minneapolis }\end{array}$ \\
& $\begin{array}{l}\text { interpretation, study } \\
\text { oversight, manuscript } \\
\end{array}$ & preparation
\end{tabular}

\begin{tabular}{|c|c|c|}
\hline $\begin{array}{l}\text { Mamatha } \\
\text { Pasnoor, } \\
\text { MD }\end{array}$ & $\begin{array}{l}\text { Department of Neurology, } \\
\text { University of Kansas } \\
\text { Medical Center, Kansas City }\end{array}$ & $\begin{array}{l}\text { Site investigator, data } \\
\text { acquisition, manuscript } \\
\text { preparation }\end{array}$ \\
\hline $\begin{array}{l}\text { Mazen M. } \\
\text { Dimachkie, } \\
\text { MD }\end{array}$ & $\begin{array}{l}\text { Department of Neurology, } \\
\text { University of Kansas } \\
\text { Medical Center, Kansas City }\end{array}$ & $\begin{array}{l}\text { Site investigator, data } \\
\text { acquisition, manuscript } \\
\text { preparation }\end{array}$ \\
\hline $\begin{array}{l}\text { Senda } \\
\text { Ajroud- } \\
\text { Driss, MD }\end{array}$ & $\begin{array}{l}\text { Department of Neurology, } \\
\text { Northwestern University, } \\
\text { Chicago, IL }\end{array}$ & $\begin{array}{l}\text { Site investigator, data } \\
\text { acquisition, manuscript } \\
\text { preparation }\end{array}$ \\
\hline $\begin{array}{l}\text { Thomas } \mathrm{H} \text {. } \\
\text { Brannagan, } \\
\text { MD }\end{array}$ & $\begin{array}{l}\text { Department of Neurology, } \\
\text { Columbia University } \\
\text { Medical Center, New York, } \\
\text { NY }\end{array}$ & $\begin{array}{l}\text { Site investigator, data } \\
\text { acquisition, manuscript } \\
\text { preparation }\end{array}$ \\
\hline $\begin{array}{l}\text { Albert A. } \\
\text { Cook, MD }\end{array}$ & $\begin{array}{l}\text { Neurology at Johns Creek, } \\
\text { LLC, Atlanta, GA }\end{array}$ & $\begin{array}{l}\text { Participants enrollment, } \\
\text { manuscript preparation }\end{array}$ \\
\hline $\begin{array}{l}\text { Timothy } \\
\text { Walton, } \\
\text { MHS, CCRP }\end{array}$ & BriovaRx, Lenexa, KS & $\begin{array}{l}\text { Protocol design, project } \\
\text { management, manuscript } \\
\text { preparation }\end{array}$ \\
\hline $\begin{array}{l}\text { Mark B. } \\
\text { Fiecas, PhD }\end{array}$ & $\begin{array}{l}\text { School of Public Heath, } \\
\text { Division of Biostatistics, } \\
\text { University of Minnesota, } \\
\text { Minneapolis }\end{array}$ & Statistical analysis \\
\hline $\begin{array}{l}\text { John T. } \\
\text { Kissel, MD }\end{array}$ & $\begin{array}{l}\text { Department of Neurology, } \\
\text { Ohio State University } \\
\text { Wexner Medical Center, } \\
\text { Columbus }\end{array}$ & $\begin{array}{l}\text { Confirmation of CIDP } \\
\text { diagnosis, manuscript } \\
\text { preparation }\end{array}$ \\
\hline
\end{tabular}

Appendix (continued)

\begin{tabular}{|c|c|c|}
\hline Name & Location & Contribution \\
\hline $\begin{array}{l}\text { Ingemar } \\
\text { Merkies, } \\
\text { MD }\end{array}$ & $\begin{array}{l}\text { Department of Neurology, } \\
\text { Maastricht University } \\
\text { Medical Centre+, the } \\
\text { Netherlands }\end{array}$ & $\begin{array}{l}\text { Protocol design, data } \\
\text { interpretation, manuscript } \\
\text { preparation }\end{array}$ \\
\hline $\begin{array}{l}\text { Kenneth C. } \\
\text { Gorson, MD }\end{array}$ & $\begin{array}{l}\text { Department of Neurology, } \\
\text { St. Elizabeth's Medical } \\
\text { Center, Tufts University } \\
\text { School of Medicine, Boston, } \\
\text { MA }\end{array}$ & $\begin{array}{l}\text { Protocol design, } \\
\text { confirmation of CIDP } \\
\text { diagnosis, data } \\
\text { interpretation, manuscript } \\
\text { preparation }\end{array}$ \\
\hline $\begin{array}{l}\text { Richard A. } \\
\text { Lewis, MD }\end{array}$ & $\begin{array}{l}\text { Department of Neurology, } \\
\text { Cedars-Sinai Medical } \\
\text { Center, Los Angeles, CA }\end{array}$ & $\begin{array}{l}\text { Protocol design, } \\
\text { confirmation of CIDP } \\
\text { diagnosis, data } \\
\text { interpretation, manuscript } \\
\text { preparation }\end{array}$ \\
\hline
\end{tabular}

\section{References}

1. Van den Bergh PY, Hadden RD, Bouche P, et al. European Federation of Neurological Societies/Peripheral Nerve Society guideline on management of chronic inflammatory demyelinating polyradiculoneuropathy: report of a joint task force of the European Federation of Neurological Societies and the Peripheral Nerve Society-first revision. Eur J Neurol 2010;17:356-363.

2. Rajabally YA, Simpson BS, Beri S, Bankart J, Gosalakkal JA. Epidemiologic variability of chronic inflammatory demyelinating polyneuropathy with different diagnostic criteria: study of a UK population. Muscle Nerve 2009;39: $432-438$.

3. Gorson KC, van Schaik IN, Merkies IS, et al. Chronic inflammatory demyelinating polyneuropathy disease activity status: recommendations for clinical research standards and use in clinical practice. J Peripher Nerv Syst 2010;15: 326-333.

4. Hughes RA, Donofrio P, Bril V, et al. Intravenous immune globulin (10\% caprylatechromatography purified) for the treatment of chronic inflammatory demyelinating polyradiculoneuropathy (ICE study): a randomised placebo-controlled trial. Lancet Neurol 2008;7:136-144.

5. Allen JA, Berger M, Querol L, Kuitwaard K, Hadden RD. Individualized immunoglobulin therapy in chronic immune-mediated peripheral neuropathies. J Peripher Nerv Syst 2018;23:78-87.

6. van Nes SI, Vanhoutte EK, van Doorn PA, et al. Rasch-Built Overall Disability Scale (R-ODS) for immune-mediated peripheral neuropathies. Neurology 2011;76: 337-345.

7. Podsiadlo D, Richardson S. The Timed "Up \& Go": a test of basic functional mobility for frail elderly persons. J Am Geriatr Soc 1991;39:142-148.

8. van Nes SI, Vanhoutte EK, Faber CG. Improving fatigue assessment in immunemediated neuropathies: the modified Rasch-Built Fatigue Severity Scale. J Peripher Nerv Syst 2009; 14:268-278.

9. Buuren SV, Groothuis-Oudshoorn K. MICE: Multivariate Imputation by Chained Equations in R. J Stat Softw 2011;45:1-67.

10. Rubin DB. Multiple Imputation for Nonresponse in Surveys. New York: John Wiley and Sons; 1987.

11. Nakagawa $S$, Schielzeth H. A general and simple method for obtaining $\mathrm{R}^{2}$ from generalized linear mixed-effects models. Methods Ecol Evol 2013;4: 133-142.

12. Merkies IS, van Nes SI, Hanna K, Hughes RA, Deng C. Confirming the efficacy of intravenous immunoglobulin in CIDP through minimum clinically important differences: shifting from statistical significance to clinical relevance. J Neurol Neurosurg Psychiatry 2010;81:1194-1199.

13. Doneddu PE, Hadden RDM. Daily grip strength response to intravenous immunoglobulin in chronic immune neuropathies. Muscle Nerve 2020;62:103-110.

14. Kuitwaard K, Fokkink WR, Brusse E, et al. Protocol of a dose response trial of IV immunoglobulin in chronic inflammatory demyelinating polyradiculneuropathy (DRIP study). J Peripher Nerv Syst 2018;23:5-10.

15. Kuitwaard K, de Gelder J, Tio-Gillen AP, et al. Pharmacokinetics of intravenous immunoglobulin and outcome in Guillain-Barré syndrome. Ann Neurol 2009;66: 597-603.

16. Fokkink W, Koch B, Ramakers C, van Doorn PA, van Gelder T, Jacobs BC. Pharmacokinetics and pharmacodynamics of intravenous immunoglobulin G maintenance therapy in chronic immune-mediated neuropathies. Clin Pharmacol Ther 2017;102: 709-716.

17. Draak TH, Pruppers MH, van Nes SI, et al. Grip strength comparison in immunemediated neuropathies: Vigorimeter vs. Jamar. J Peripher Nerv Sys 2015;20:269-276. 
18. Vanhoutte EK, Latov N, Deng C, et al. Vigorimeter grip strength in CIDP: a responsive tool that rapidly measures the effect of IVIG: the ICE study. Eur J Neurol 2013;20:748-755.

19. Beaton DE, Boers M, Wells GA. Many faces of the minimal clinically importan difference (MCID): a literature review and directions for future research. Curr Opin Rheumatol 2002;14:109-114.

20. Allen JA, Merkies ISJ, Lewis RA. Monitoring clinical course and treatment response in chronic inflammatory demyelinating polyneuropathy during routine care: a review of clinical and laboratory assessment measures. JAMA Neurol 2020;77:1159-1166.
21. Hughes R, Bensa S, Willison $\mathrm{H}$, et al; Inflammatory Neuropathy Cause and Treatment (INCAT) Group. Randomized controlled trial of intravenous immunoglobulin versus oral prednisolone in chronic inflammatory demyelinating polyradiculoneuropathy. Ann Neurol 2001;50:195-201.

22. Allen JA, Lewis RA. CIDP diagnostic pitfalls and perception of treatment benefit. Neurology 2015;85:498-504

23. Kuitwaard K, van Doorn PA, Vermeulen M, et al. Serum IgG levels in IV immunoglobulin treated chronic inflammatory demyelinating polyneuropathy. J Neurol Neurosurg Psych 2013;84:859-861. 


\section{Neurology}

Quantifying Treatment-Related Fluctuations in CIDP: Results of the GRIPPER Study Jeffrey A. Allen, Mamatha Pasnoor, Mazen M. Dimachkie, et al.

Neurology 2021;96;e1876-e1886 Published Online before print February 16, 2021

DOI 10.1212/WNL.0000000000011703

This information is current as of February 16, 2021

Updated Information \&
Services
References
Citations
Subspecialty Collections

Permissions \& Licensing

Reprints including high resolution figures, can be found at: http://n.neurology.org/content/96/14/e1876.full

This article cites 22 articles, 3 of which you can access for free at: http://n.neurology.org/content/96/14/e1876.full\#ref-list-1

This article has been cited by 1 HighWire-hosted articles: http://n.neurology.org/content/96/14/e1876.full\#\#otherarticles

This article, along with others on similar topics, appears in the following collection(s):

\section{All Clinical trials}

http://n.neurology.org/cgi/collection/all_clinical_trials

Chronic inflammatory demyelinating polyneuropathy

http://n.neurology.org/cgi/collection/chronic_inflammatory_demyelinat ing_polyneuropathy

Outcome research

http://n.neurology.org/cgi/collection/outcome_research

Peripheral neuropathy

http://n.neurology.org/cgi/collection/peripheral_neuropathy

Information about reproducing this article in parts (figures,tables) or in its entirety can be found online at:

http://www.neurology.org/about/about_the_journal\#permissions

Information about ordering reprints can be found online:

http://n.neurology.org/subscribers/advertise

Neurology ${ }^{\circledR}$ is the official journal of the American Academy of Neurology. Published continuously since 1951, it is now a weekly with 48 issues per year. Copyright (C 2021 American Academy of Neurology. All rights reserved. Print ISSN: 0028-3878. Online ISSN: 1526-632X.

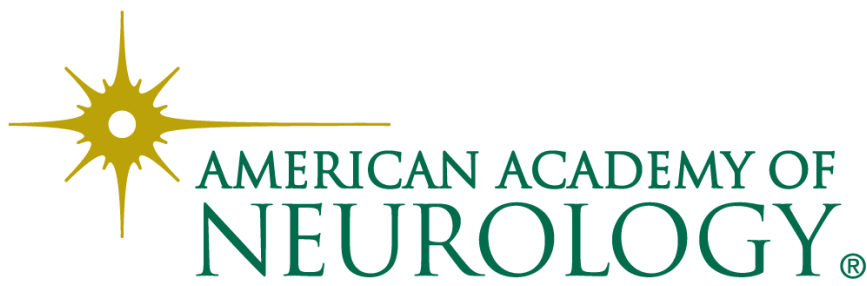

